\title{
A novel tablet computer platform for advanced language mapping during awake craniotomy procedures
}

\author{
Melanie A. Morrison, BSc, ${ }^{1,2}$ Fred Tam, BMath, ${ }^{1}$ Marco M. Garavaglia, MD, ${ }^{5}$ \\ Laleh Golestanirad, PhD, ${ }^{6}$ Gregory M. T. Hare, MD, PhD, ${ }^{3,5}$ Michael D. Cusimano, MD, PhD, ${ }^{3,4}$ \\ Tom A. Schweizer, PhD, ${ }^{3}$ Sunit Das, MD, PhD, ${ }^{3,4}$ and Simon J. Graham, PhD, PEng ${ }^{1,2}$ \\ ${ }^{1}$ Physical Sciences, Sunnybrook Research Institute, Toronto; ${ }^{2}$ Department of Medical Biophysics, University of Toronto; ${ }^{3}$ Keenan \\ Research Centre, ${ }^{4}$ Division of Neurosurgery, and ${ }^{5}$ Department of Anesthesia, St. Michael's Hospital, Toronto, Ontario, Canada; \\ and ${ }^{6}$ Athinoula A. Martinos Center for Biomedical Imaging, Harvard Medical School, Boston, Massachusetts
}

\begin{abstract}
A computerized platform has been developed to enhance behavioral testing during intraoperative language mapping in awake craniotomy procedures. The system is uniquely compatible with the environmental demands of both the operating room and preoperative functional MRI (fMRI), thus providing standardized testing toward improving spatial agreement between the 2 brain mapping techniques. Details of the platform architecture, its advantages over traditional testing methods, and its use for language mapping are described. Four illustrative cases demonstrate the efficacy of using the testing platform to administer sophisticated language paradigms, and the spatial agreement between intraoperative mapping and preoperative fMRI results. The testing platform substantially improved the ability of the surgeon to detect and characterize language deficits. Use of a written word generation task to assess language production helped confirm areas of speech apraxia and speech arrest that were inadequately characterized or missed with the use of traditional paradigms, respectively. Preoperative fMRI of the analogous writing task was also assistive, displaying excellent spatial agreement with intraoperative mapping in all 4 cases. Sole use of traditional testing paradigms can be limiting during awake craniotomy procedures. Comprehensive assessment of language function will require additional use of more sophisticated and ecologically valid testing paradigms. The platform presented here provides a means to do so.

http://thejns.org/doi/abs/10.3171/2015.4.JNS15312
\end{abstract}

KEY WORDS brain mapping; brain tumor; functional neurosurgery; glioma; language; surgery; tablet computing; surgical technique

I NTRAOPERATIVE language mapping during awake craniotomy to facilitate tumor resection remains challenging. The selection of intraoperative tasks can have a large impact on the identification of critical language areas by the gold standard intraoperative brain mapping approach, direct cortical electrical stimulation (DCES)., 2,15 Number counting and visual object naming tasks are typically used to assess speech articulation and semantic and lexical retrieval, respectively. ${ }^{1,3,5,11,12,17,18,21}$ These traditional tasks are advantageous for their simplicity, speed, and accuracy in detecting language errors, but are limited in their cognitive demand and ecological validity (i.e., their ability to elicit responses that represent the complexities of everyday behavior) ${ }^{20} \mathrm{~A}$ more comprehensive assessment of language function requires the integration of more so- phisticated intraoperative task batteries and ecologically valid assessment paradigms. Such task batteries should also be flexible, allowing the inclusion or exclusion of tests, according to patient-specific characteristics, such as tumor location, or individual anatomofunctional correlations that may be revealed by presurgical functional neuroimaging. Regarding the latter consideration, preoperative functional MRI (fMRI) has emerged as a very useful method to provide brain maps for neurosurgical planning. ${ }^{23,24}$ The benefit of fMRI as a predictive tool for the operating room inherently depends on agreement between the brain mapping techniques of fMRI and DCES, which in turn would benefit from the development of mature standardized testing paradigms that could translate functionally and practically between the 2 environments.

ABBREVIATIONS DCES = direct cortical electrical stimulation; DVR = digital video recorder; fMRI = functional MRI; IV = intravenous; $L C D=$ liquid crystal display; $S O P=$ standard operating procedure. 
In response to these needs, we have developed a flexible, computerized platform for behavioral testing to map brain function during preoperative fMRI and DCES. Given the increasingly wide use of touch-sensitive tablet computing technology in society, we have based our testing platform around tablet capabilities and interactions. The platform is a natural extension of our previous work to develop a tablet-based solution exclusively for fMRI environments. ${ }^{22}$ Whereas fMRI of overt speech is prone to signal artifacts, ${ }^{9,10}$ writing is an ecologically valid form of language production that can be readily recorded, assessed, and quantified using a tablet, yielding robust fMRI data quality. In this technology report, we describe enhanced tablet testing platform architecture and demonstrate the utility of the tablet platform in writing tasks for language mapping with DCES. Successful application of the platform is demonstrated through 4 illustrative cases, each involving preoperative fMRI language mapping and intraoperative DCES language mapping during an awake craniotomy for glioma resection.

\section{Intraoperative Testing Platform Architecture}

Figure 1 shows a schematic overview of the testing platform, together with images of the main components used in a working prototype, implemented in an operating room at St. Michael's Hospital in Toronto, Ontario, Canada. Informed consent was obtained for each patient, and all patient testing was performed with approval from the Hospital's Research Ethics Board. The core of the testing platform consisted of an fMRI-compatible tablet, ${ }^{22}$ including a touch-sensitive tablet surface with an optional writing stylus; a controller box that powered the tablet and transmitted behavioral data recorded during tablet interactions in real time; and a stimulus computer with flexible software to program and execute diverse behavioral tests, receive and quantify tablet data, and provide testrelated feedback to the patient and the neurosurgical team (E-Prime, Psychology Software Tools). ${ }^{22}$ Behavioral testing was administered by a member of the research team (M.A.M.) who initiated the computer software on the stimulus computer, as directed by the surgeon.

During preoperative fMRI, the patient must undergo behavioral testing with restricted head motion and visibility while lying within the confined bore of an MRI system. When the fMRI-compatible tablet system was designed, these constraints made it necessary to depart from the modern mode of tablet computing that involves use of an integrated touch-sensitive visual display. Instead, separate hardware pathways were implemented for visual stimulus presentation and tablet interactions. Patients were presented with visual stimuli and visual representation of stylus responses, which were transmitted using a liquid crystal display (LCD) projector onto a rear-projection screen, viewed through an angled mirror. Tablet interactions were conducted with the touch-sensitive surface mounted over the torso. Analogous pathways were developed for the intraoperative testing platform to accommodate constraints faced by patients during awake craniotomy (i.e., head immobilization, and confined positioning on an operating table under surgical drapes). To facilitate behavioral testing, visual stimuli and tablet interactions were displayed intraoperatively for the patient by using a 5-inch LCD display (Ikan VL5).

Additional design criteria were incorporated into the prototype testing platform to enhance utility during intraoperative DCES, considering the needs of the patient (adjustability for patient size, position, comfort, and ease of interaction), the neurosurgeon (robust time-efficient implementation, and ease of interaction with the patient, testing platform, and surgical team), and the anesthesiologist and nursing staff (minimal footprint of the testing platform in the operating room, minimal patient obstruction, minimal setup and teardown). Consequently, in addition to the tablet, the stimulus computer, and the patient display described above, the platform included additional video cameras for monitoring the patient's face (Swann ADS120), hand (Swann PRO-642), foot (Swann PRO-642), and brain (Swann PRO-642) during DCES; adjustable support arms, brackets, and clamps for mounting the cameras; a medical power isolation transformer (Dale Technology IT400); a video splitter (Rocketfish HDMI Splitter RFG1182) and converter (StarTech HD2VID); and a 9-channel digital video recorder (DVR; Swann DVR9-4200 960H) and 21-inch ceiling-mounted LCD surgical display (Sony LMD-2140MD). Lastly, the equipment was housed in a portable compact equipment cart (Anthro Trolley), and stationed on the anesthesia side of the operating room during surgery. Tablet hardware, including the stimulus computer, was fixed on the top level of the cart; the video hardware on the middle level; and the medical power isolation transformer, along with all device power supplies, on the bottom level. The cameras, support arms, brackets, and clamps were stored in a drawer under the middle level such that they were easily accessible for setup.

Following anesthesia induction and patient positioning, the tablet, patient display, and cameras were mounted in the appropriate locations. For rapid, flexible deployment, the equipment setup was predetermined by a standard operating procedure (SOP) prescribed for each patient before surgery. Table 1 provides the SOP details for 5 different patient scenarios, according to the surgical procedure, the subsequent patient positioning, and the dominant writing hand. Overall, the setup procedure remained similar for each patient scenario, requiring only small adjustments. Total setup time was approximately 15 minutes, undertaken following application of the surgical drapes in parallel with the craniotomy procedure, such that the platform was fully operational when the surgeon was ready to perform intraoperative mapping. No significant surgical time was added to the procedure through setup or use of the testing platform.

Figure 1 (left) schematically shows a case in which supine positioning was used with the patient's head turned to the right. The brain camera (Fig. 1A, item 1) was mounted on 1 of 2 ceiling-mounted surgical lamps above the surgeon's head, providing a clear view of the surgical field. The patient display (Fig. 1B, item 2) with mounted face camera (Fig. 1B, item 3) was positioned for comfortable viewing, and was mounted to a Mayo stand near the patient's head. The tablet (Fig. 1C, item 4) was mounted to the main intravenous (IV) pole at a comfortable writing position, along with a camera to record hand movements (Fig. 1C, item 5). Lastly, a camera mounted to a second 

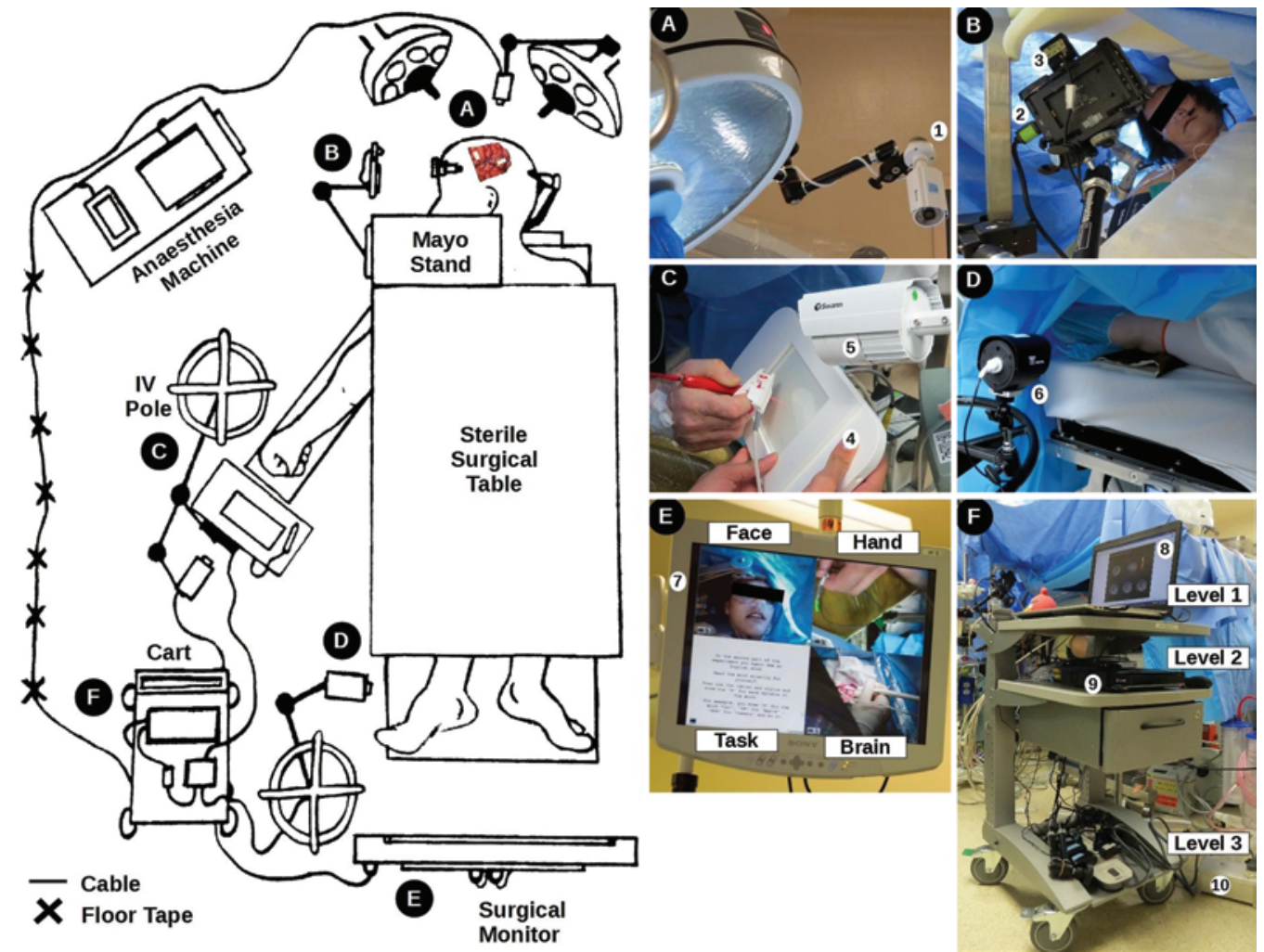

FIG. 1. Left: Schematic drawing of the operating room configuration with the intraoperative testing platform. Extra surgical equipment has been omitted from this drawing for clarity. Right: Parts of the intraoperative testing platform corresponding to sites $A-F$ labeled in the schematic. Individual items are labeled 1-10. A = surgical field: brain camera (1); $B=$ site of patient interactions with visual stimuli: patient display (2), mounted face camera (3); $C=$ site of patient interactions with the tablet system: tablet (4), camera to record hand movements (5); $D=$ foot end of the surgical table: camera to record foot movements $(6) ; E=$ site where the video monitoring system is stationed: $L C D$ surgical display (7); $F=$ site where the intraoperative testing platform is stationed: stimulus computer (8), DVR (9), medical power isolation transformer (10). Figure is available in color online only.

IV pole at the far end of the operating table recorded foot movements (Fig. 1D, item 6).

The final component of the intraoperative testing platform was the video monitoring system, which was configured by connecting output from the DVR (Fig. 1F, item 9) to the LCD surgical display (Fig. 1E, item 7) that was suspended over the foot of the operating table. With the medical power isolation transformer (Fig. 1F, item 10) plugged into the nearest outlet, the LCD surgical split-screen display presented the surgical team with real-time video access to the patient's face, hand, visual stimuli, and tablet responses for the selected task from the stimulus computer (Fig. 1F, item 8) and surgical field. Patient responses (e.g., yes or no decisions, written responses) were also updated on the patient display and video monitor for visualization by the patient and test administrator, respectively. Examples of written behavioral responses are shown in Fig. 2 and described in more detail below.

\section{Illustrative Cases}

\section{Characterizing Tongue and Speech Apraxia}

A 53-year-old right-handed woman (Case 1) underwent a repeat right frontotemporal awake craniotomy following glioblastoma recurrence, resulting in mild left-sided weakness and dysarthria. The anesthetic approach for this surgery (and for subsequent cases) included an initial bupivacaine-based scalp nerve block and use of dexmedetomidine as the primary anesthetic agent. ${ }^{7}$ Intraoperative mapping was initiated with a traditional number counting task. Stimulating at $2 \mathrm{~mA}$, a broad area was demarcated where inconsistent dysarthric speech was observed with varying levels of speech arrest. Tongue movement was assessed while stimulating at $1 \mathrm{~mA}$ and $2 \mathrm{~mA}$, but was equivocal. The intraoperative testing platform was then used to administer a tablet-based word generation task (phonemic fluency; Fig. 2) involving the presentation of a letter and 60 seconds for the patient to think of and write words beginning with the letter. The task was executed without difficulty; however, when repeated in combination with overt speech ("tell us the word(s) you are writing"), the patient was unable to communicate the words verbally. The patient described her deficit as tongue clumsiness, despite the ability to move the tongue on command, think of words, and write words during stimulation of the same area. Based on these findings, tongue apraxia was concluded rather than aphasic speech arrest. The stimulation site was marked accordingly (Fig. 3A), agreeing well with preoperative fMRI data obtained during tongue movement (Fig. 3B).

Similar findings were observed for a 23-year-old right-handed woman (Case 2) who underwent a left fron- 
TABLE 1. SOP details for equipment setup based on a righthanded patient in supine and lateral orientations dictated by the tumor location*

\begin{tabular}{|c|c|}
\hline Patient Position & SOP Details \\
\hline \multicolumn{2}{|l|}{ Supine } \\
\hline Head neutral (a) & $\begin{array}{l}\text { Anesthesia equipment on rt side. } \\
\text { Intraop testing platform on rt side. } \\
\text { Rt arm board, It arm secured. } \\
\text { 2nd IV pole, 2nd sterile drape on rt side. } \\
\text { Mayo stand on right side, elevated for better } \\
\quad \text { access to patient's face. }\end{array}$ \\
\hline Head turned rt (b)† & $\begin{array}{l}\text { Anesthesia equipment on rt side. } \\
\text { Intraop testing platform on rt side. } \\
\text { Rt arm board, It arm secured. } \\
\text { 2nd IV pole, 2nd sterile drape on rt side. } \\
\text { Mayo stand on rt side. }\end{array}$ \\
\hline Head turned It (c) & $\begin{array}{l}\text { Anesthesia equipment on rt side. } \\
\text { Intraop testing platform on rt side. } \\
\text { Lt arm board. } \\
\text { Rt shoulder propped and rt arm extended to It } \\
\text { side for writing. } \\
\text { 2nd IV pole, 2nd sterile drape on rt side. } \\
\text { Mayo stand on It side. }\end{array}$ \\
\hline \multicolumn{2}{|l|}{ Lateral } \\
\hline Lt lateral (d) & $\begin{array}{l}\text { Anesthesia equipment on It side. } \\
\text { Intraop testing platform on It side. } \\
\text { Stacked arm boards. } \\
\text { 2nd IV pole, 2nd sterile drape on It side. } \\
\text { Mayo stand on It side. }\end{array}$ \\
\hline Rt lateral (e) & $\begin{array}{l}\text { Anesthesia equipment on rt side. } \\
\text { Intraop testing platform on rt side. } \\
\text { Stacked arm boards. } \\
\text { Rt forearm propped for writing. } \\
\text { 2nd IV pole, 2nd sterile drape on rt side. } \\
\text { Mayo stand on rt side. }\end{array}$ \\
\hline
\end{tabular}

* Opposite for left-handedness.

$\dagger$ Refer to Fig. 1.

totemporal awake craniotomy following diagnosis of a low-grade insular glioma, resulting in frequent seizures. Stimulation during a number-counting task caused aphasic speech interruption, despite the patient's ability to move her tongue on command and to use the intraoperative testing platform to perform a word generation task by overt speech. The area originally labeled as speech arrest was recharacterized as speech apraxia (Fig. 3C), agreeing well with preoperative fMRI data obtained during tongue movement (Fig. 3D).

\section{Detecting Speech Arrest}

A 43-year-old left-handed man (Case 3) underwent a left frontotemporal awake craniotomy following diagnosis of glioblastoma, resulting in headaches and speech difficulty. Intraoperative mapping began with a traditional number-counting task that was executed without difficulty when stimulating up to $4 \mathrm{~mA}$. The patient was engaged in conversational speech and asked to recite the song "Happy Birthday;" however, no deficits were observed with stimu- lation up to $4 \mathrm{~mA}$. The word-generation task was then administered using the intraoperative testing platform with the patient responding by overt speech. Stimulation at 2 $\mathrm{mA}$ localized an area of speech arrest with task interruption (Fig. 4A) that had not been identified using traditional paradigms. Preoperative mapping of the analogous task by fMRI of written responses produced activation maps that agreed well with our intraoperative mapping results (Fig. 4B).

A similar finding was observed in a 38-year-old righthanded woman (Case 4) who underwent a right frontal awake craniotomy following diagnosis of a low-grade frontal glioma, resulting in frequent seizures. Number counting and general speech were unaffected by stimulation, but the intraoperative testing platform identified 2 areas where stimulation induced speech arrest and task interruption while the patient performed the word generation task with overt speech (Fig. 4C). Again, our intraoperative findings confirmed fMRI predictions based on written responses, showing excellent spatial agreement between both brain mapping procedures (Fig. 4D).

\section{Discussion}

Intraoperative language mapping during awake craniotomy by DCES has been critical to mediating the competing goals of aggressive tumor resection and maintenance of neurological function in patients with brain tumors. There is further scope to enhance the utility of DCES by considering the choice of behavioral tasks used during surgery ${ }^{4}$ for improved detection and characterization of language deficits, and by using preoperative functional neuroimaging data during formulation of the surgical plan. ${ }^{6}$ The present work describes our initial attempt to develop a flexible behavioral testing platform that allows comprehensive language testing in a standardized fashion during both preoperative fMRI and intraoperative DCES.

In designing our testing platform, we provided the surgeon with the capability to map brain function with a battery of tasks, ranging from simple assessments (e.g., number counting) to more sophisticated and ecologically valid tests of language processing (e.g., phonemic fluency ${ }^{9,14}$ ), involving written output. Sequential use of DCES during both written and overt response modes can potentially assist in determining the functional roles of cortical language areas.

The computerized testing platform provides a highly flexible environment for developing a wide variety of language assessments, which can be implemented and validated off-line with the expertise of a neuropsychologist or speech language pathologist. Although the present work illustrates the system capabilities specifically for language assessment during DCES mapping of patients with brain tumors, tasks can be designed to assess different aspects of mental processing, and the testing platform is also applicable for use in other types of functional brain surgery (e.g., epilepsy, electrode placement for deep brain stimulation). The user interface for the platform is simple, and the use of automated behavioral tasks allows for easy adjustment of task parameters to meet the needs of the patient or surgeon during intraoperative testing (e.g., increase font size, reduce stimulus presentation time). 


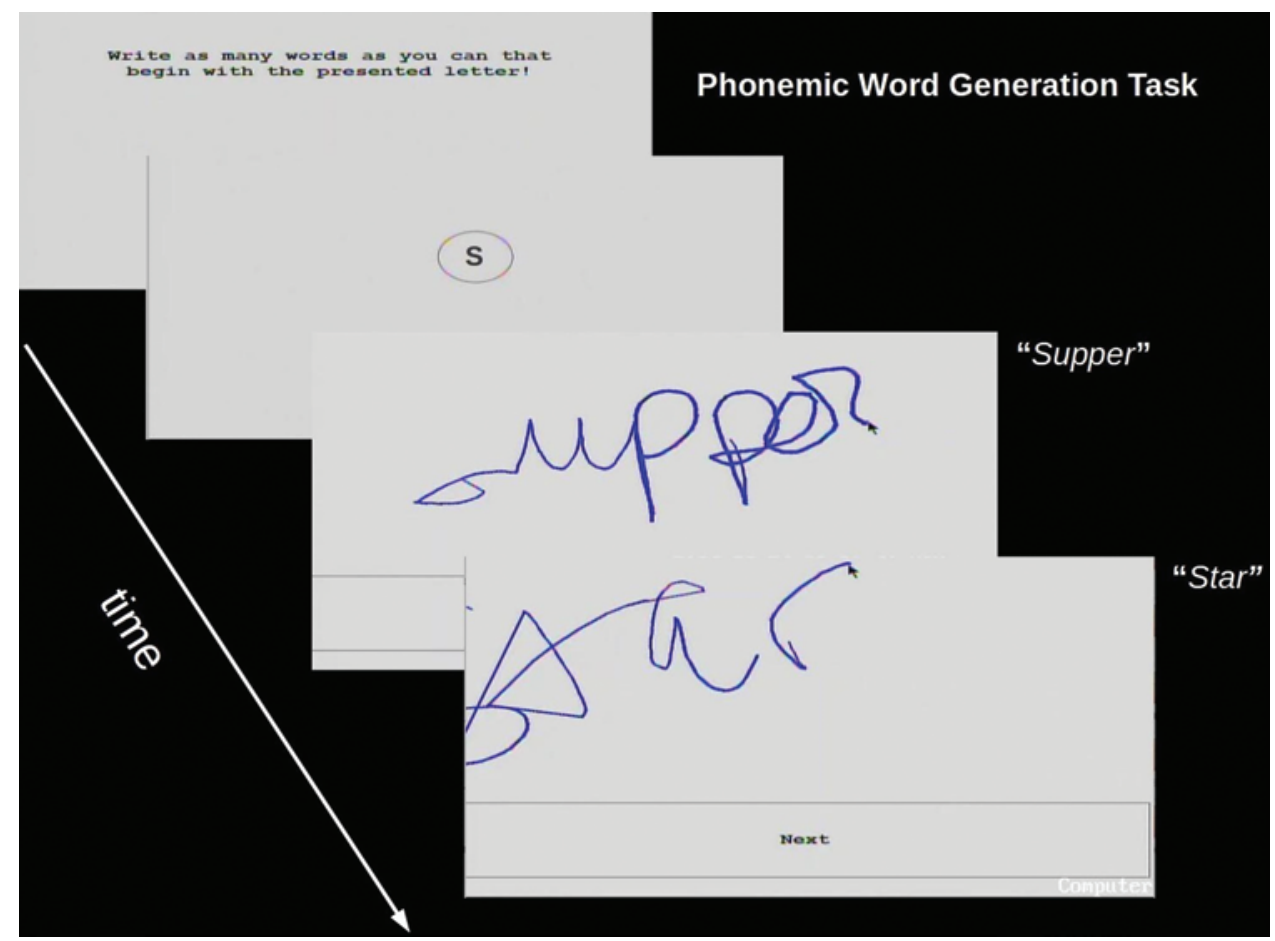

FIG. 2. Visual stimuli and task-related feedback for a phonemic fluency word-generation task. The words "supper" and "star" were generated for the letter "S." Figure is available in color online only.

The video monitoring system is a particularly important component of the testing platform, as it allows the surgical team to monitor patient status directly in real time during the awake phase of surgery, and especially during behavioral assessment. For the surgeon applying DCES, video monitoring eliminates any need for a "middle man" to report DCES effects on patient performance. Instead, DCES can be applied with real-time visual feedback of patient effects to the surgeon, as well as other members of the surgical team, for enhanced team communication and joint evaluation of the patient during behavioral testing. This not only improves efficiency but also enables DCES mapping to be undertaken during behavioral tasks that were previously impractical (e.g., double tasks, ${ }^{6}$ comprehensive writing tasks).

To our knowledge, only 1 other group has provided a focused report of a computerized platform for behavioral testing and monitoring during intraoperative mapping. ${ }^{25}$ Important differences exist between this prior development, conducted in Japan, and the platform described in the present work. Unlike the Japanese platform, our platform is portable and was designed to enable flexible, computerized administration of both simple and sophisticated language paradigms, including use of tablet technology in both the operating room and during preoperative fMRI to perform standardized testing.

Our case experiences demonstrate the efficacy of our testing platform for administering sophisticated language paradigms, to help differentiate language deficits during DCES, and to identify cortical areas with critical roles in language processing. In 2 cases presented (Cases 1 and 2) a traditional counting task misled us to believe that DCES had produced speech arrest in a particular location, when,
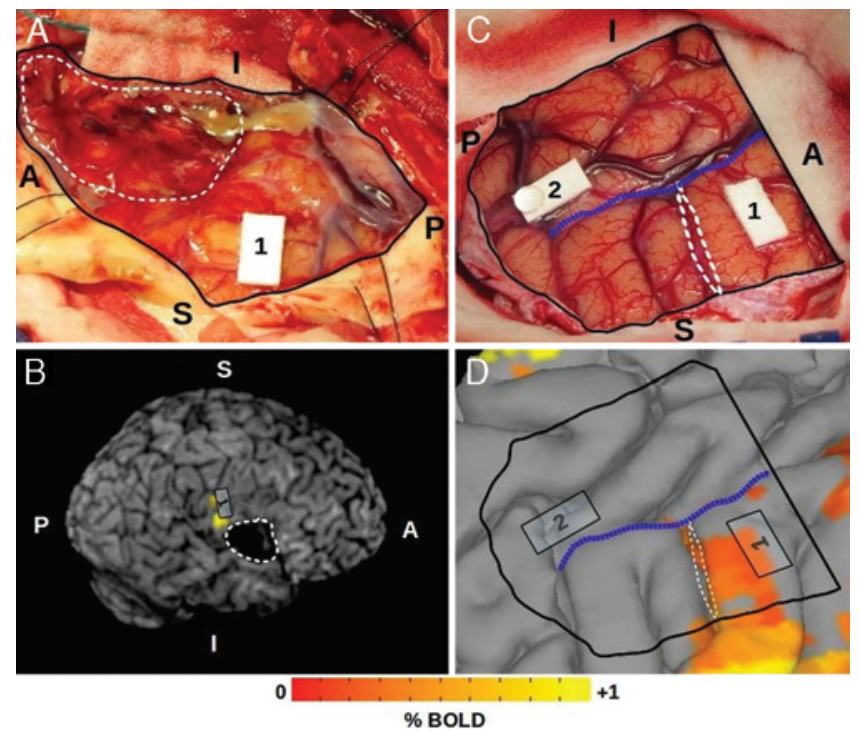

FIG. 3. Case 1. A. Image of craniotomy surface, with speech apraxia site marked at the lower postcentral gyrus (chip \#1). The solid black line outlines the craniotomy extent. The white dashed line outlines the tumor/ resection cavity. I, P, S, and A correspond to inferior, posterior, superior, and anterior directions, respectively. B. Three-dimensional reconstruction of preoperative fMRI results for a tongue movement task. The position of chip \#1 is overlaid, corresponding to the approximate location of speech apraxia finding. C. Case 2. Image of craniotomy surface, with speech apraxia site marked at the lower precentral gyrus (chip \#1). Chip \#2 approximates Wernicke's area and was additionally mapped using a word-copying task. The white dashed line outlines the entry point to the deep-seated tumor. The line of blue dashes marks the location of the Sylvian fissure. D. Case 2. Intraoperative mapping data superimposed on a $3 D$ reconstruction of preoperative fMRI results for a tongue movement task. Figure is available in color online only. 


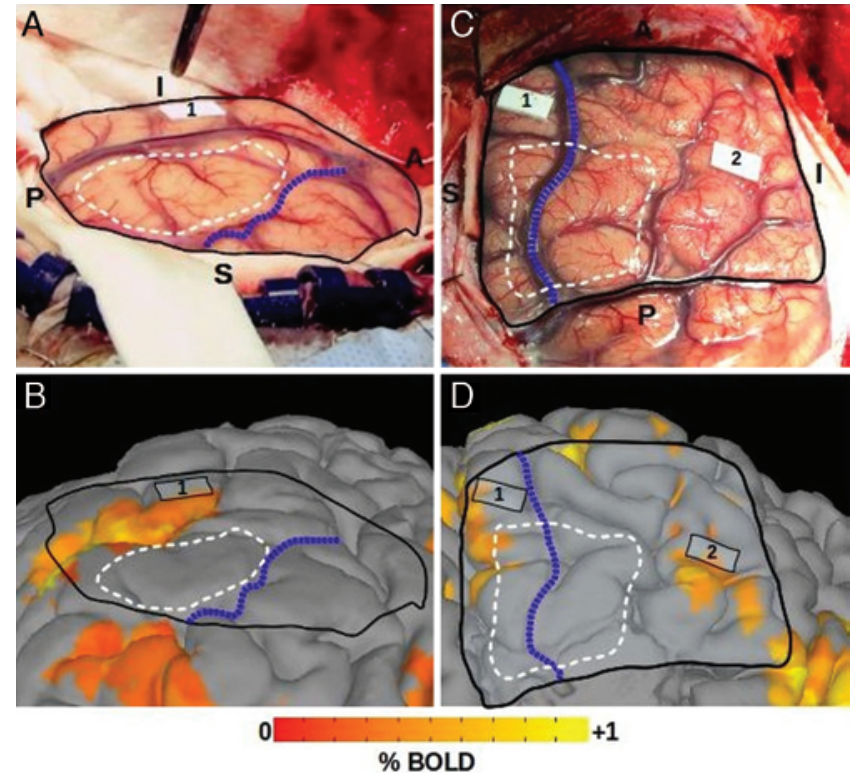

FIG. 4. Case 3. A. Image of craniotomy surface, with chip \#1 marking the site of speech arrest on the posterior end of the middle temporal gyrus. The solid black line outlines the extent of the craniotomy. The white dashed line outlines the tumor/resection cavity. The blue dashed line marks the location of the sylvian fissure. I, A, S, and P correspond to inferior, anterior, superior, and posterior directions, respectively. B. Chip \#1 corresponding to the site of speech arrest overlaid on the $3 \mathrm{D}$ reconstruction of preoperative fMRI results for the wordgeneration task. C. Case 4. Chips \#1 and \#2 marking sites of speech arrest mapped on the superior and inferior frontal gyri, respectively. The line of blue dashes marks the location of a large, superficial blood vessel. D: Case 4. Intraoperative mapping data superimposed on a 3D reconstruction of preoperative fMRI results for the word-generation task. Figure is available in color online only.

as made apparent during more sophisticated testing with the platform, tongue or speech apraxia was the correct characterization. Because the literature suggests that recovery from speech apraxia is better than recovery from aphasia ${ }_{13}^{13}$ this finding had a direct immediate effect on the surgeon's decision to carry forward with further resection. Two additional cases (Cases 3 and 4) illustrate the use of our testing platform to improve the detection of cortical language areas, in circumstances where traditional testing paradigms were not sensitive. Overall, these cases support our assertion that benefits are provided by the use of sophisticated paradigms to assess language more comprehensively during DCES.

Our results also suggest that sole reliance on overt speech responses is a potential limitation in traditional DCES language testing paradigms and support previous finding ${ }^{16,19}$ that preoperative fMRI data can accurately predict intraoperative findings and play an important assistive role in optimizing DCES procedures and surgical decision making. Others have reported in more detail on the promising spatial agreement between preoperative fMRI and DCES results, although such work has been confounded by lack of standardized behavioral testing that bridges the 2 techniques. ${ }^{8}$ By using the tablet technology as a standardized testing platform, we hope to move toward administration of very similar tasks during fMRI and DCES, thus potentially enhancing the spatial agreement between fMRI and DCES. For example, the word generation task requires written tablet responses during fMRI to prevent motion artifacts, but can be evaluated during DCES with either written or overt responses. Although the tasks may not be "identical," they are very similar and the literature has shown substantial commonality between the 2 response modes. ${ }^{9}$ Qualitative evaluation of our preliminary data is very promising and our collective results, augmented by additional patient recruitment, will be the subject of a report by our group in the immediate future.

\section{Conclusions}

A flexible platform for behavioral testing has been developed for use with intraoperative DCES during awake craniotomy. The platform, which includes an interactive tablet interface, enables behavioral testing to be standardized between preoperative fMRI and intraoperative DCES to enhance the spatial agreement between the 2 methods and provide enhanced value in surgical decision making. The testing platform is particularly advantageous for the administration of more sophisticated tasks for mapping language function, including use of written in addition to spoken responses. The utility of the platform was demonstrated in 4 illustrative cases, in whom better detection and characterization of language was achieved compared with the use of more conventional testing, with excellent spatial agreement between preoperative fMRI and intraoperative DCES results. Application of the tablet platform to a larger group of brain cancer patients will be reported in the future.

\section{Acknowledgments}

The authors thank Dr. Andrea Rigamonti, Shannon Milburn, and Forough Kalani for their assistance during the awake craniotomy procedures. This work was supported by a Canadian Cancer Society operating grant. Dr. Hare has received support from a merit award issued by the Department of Anesthesia at the University of Toronto.

\section{References}

1. Benzagmout M, Gatignol P, Duffau H: Resection of World Health Organization Grade II gliomas involving Broca's area: methodological and functional considerations. Neurosurgery 61:741-753, 2007

2. Berger MS, Ojemann GA: Intraoperative brain mapping techniques in neuro-oncology. Stereotact Funct Neurosurg 58:153-161, 1992

3. De Benedictis A, Moritz-Gasser S, Duffau H: Awake mapping optimizes the extent of resection for low-grade gliomas in eloquent areas. Neurosurgery 66:1074-1084, 2010

4. De Witte E, Mariën P: The neurolinguistic approach to awake surgery reviewed. Clin Neurol Neurosurg 115:127145, 2013

5. Duffau H, Peggy Gatignol ST, Mandonnet E, Capelle L, Taillandier L: Intraoperative subcortical stimulation mapping of language pathways in a consecutive series of 115 patients with Grade II glioma in the left dominant hemisphere. J Neurosurg 109:461-471, 2008

6. Fernández Coello A, Moritz-Gasser S, Martino J, Martinoni M, Matsuda R, Duffau H: Selection of intraoperative tasks 
for awake mapping based on relationships between tumor location and functional networks. J Neurosurg 119:13801394,2013

7. Garavaglia MM, Das S, Cusimano MD, Crescini C, Mazer CD, Hare GMT, et al: Anesthetic approach to high-risk patients and prolonged awake craniotomy using dexmedetomidine and scalp block. J Neurosurg Anesthesiol 26:226-233, 2014

8. Giussani C, Roux FE, Ojemann J, Sganzerla EP, Pirillo D, Papagno C: Is preoperative functional magnetic resonance imaging reliable for language areas mapping in brain tumor surgery? Review of language functional magnetic resonance imaging and direct cortical stimulation correlation studies. Neurosurgery 66:113-120, 2010

9. Golestanirad L, Das S, Schweizer TA, Graham SJ: A preliminary fMRI study of a novel self-paced written fluency task: observation of left-hemispheric activation, and increased frontal activation in late vs. early task phases. Front Hum Neurosci 9:113, 2015

10. Gracco VL, Tremblay P, Pike B: Imaging speech production using fMRI. Neuroimage 26:294-301, 2005

11. Grundy PL, Weidmann C, Bernstein M: Day-case neurosurgery for brain tumours: the early United Kingdom experience. Br J Neurosurg 22:360-367, 2008

12. Gupta DK, Chandra PS, Ojha BK, Sharma BS, Mahapatra AK, Mehta VS: Awake craniotomy versus surgery under general anesthesia for resection of intrinsic lesions of eloquent cortex--a prospective randomised study. Clin Neurol Neurosurg 109:335-343, 2007

13. Kertesz A, McCabe P: Recovery patterns and prognosis in aphasia. Brain 100:1-18, 1977

14. Lurito JT, Kareken DA, Lowe MJ, Chen SH, Mathews VP: Comparison of rhyming and word generation with FMRI. Hum Brain Mapp 10:99-106, 2000

15. Mandonnet E: Intraoperative electrical mapping: advances, limitations and perspectives, in Duffau H (ed): Brain Mapping: From Neural Basis of Cognition to Surgical Applications. Vienna: Springer, 2011, pp 101-106

16. Meier MP, Ilmberger J, Fesl G, Ruge MI: Validation of functional motor and language MRI with direct cortical stimulation. Acta Neurochir (Wien) 155:675-683, 2013

17. Parney IF, Goerss SJ, McGee K, Huston J III, Perkins WJ, Meyer FB: Awake craniotomy, electrophysiologic mapping, and tumor resection with high-field intraoperative MRI. World Neurosurg 73:547-551, 2010

18. Rofes A, Miceli G: Language mapping with verbs and sentences in awake surgery: a review. Neuropsychol Rev 24:185-199, 2014

19. Roux FE, Boulanouar K, Lotterie JA, Mejdoubi M, LeSage
JP, Berry I: Language functional magnetic resonance imaging in preoperative assessment of language areas: correlation with direct cortical stimulation. Neurosurgery 52:13351347,2003

20. Serletis D, Bernstein M: Prospective study of awake craniotomy used routinely and nonselectively for supratentorial tumors. J Neurosurg 107:1-6, 2007

21. Talacchi A, Santini B, Savazzi S, Gerosa M: Cognitive effects of tumour and surgical treatment in glioma patients. J Neurooncol 103:541-549, 2011

22. Tam F, Churchill NW, Strother SC, Graham SJ: A new tablet for writing and drawing during functional MRI. Hum Brain Mapp 32:240-248, 2011

23. Tieleman A, Deblaere K, Van Roost D, Van Damme O, Achten E: Preoperative fMRI in tumour surgery. Eur Radiol 19:2523-2534, 2009

24. Vlieger EJ, Majoie CB, Leenstra S, Den Heeten GJ: Functional magnetic resonance imaging for neurosurgical planning in neurooncology. Eur Radiol 14:1143-1153, 2004

25. Yoshimitsu K, Suzuki T, Muragaki Y, Chernov M, Iseki H: Development of modified intraoperative examination monitor for awake surgery (IEMAS) system for awake craniotomy during brain tumor resection. Conf Proc IEEE Eng Med Biol Soc 2010:6050-6053, 2010

\section{Disclosures}

Dr. Simon Graham and Fred Tam have been issued a patent for the invention of the tablet technology. The primary author Melanie Morrison and coauthors Dr. Simon Graham, Dr. Tom Schweizer, Dr. Sunit Das, and Fred Tam, have just filed a provisional US patent application covering use of the tablet system in neurosurgery.

\section{Author Contributions}

Conception and design: Morrison, Tam. Acquisition of data: Morrison. Analysis and interpretation of data: Morrison, Das. Drafting the article: Morrison. Critically revising the article: Morrison, Tam, Cusimano, Schweizer, Das, Graham. Reviewed submitted version of manuscript: all authors. Approved the final version of the manuscript on behalf of all authors: Morrison. Statistical analysis: Morrison. Administrative/technical/material support: all authors. Study supervision: Das, Graham.

\section{Correspondence}

Melanie Morrison, 605 S-Wing, 2075 Bayview Ave., Toronto, ON M4N 3M5, Canada. email: melanie.morrison@hotmail.com. 